\title{
Precision Tiltmeter as a Reference for Slope Measuring Instruments
}

\author{
Jonathan L. Kirschman, Edward E. Domning, Gregory Y. Morrison, \\ Brian V. Smith, and Valeriy V. Yashchuk \\ Lawrence Berkeley National Laboratory, Berkeley, California, 94720
}

\begin{abstract}
The next generation of synchrotrons and free electron lasers require extremely high-performance $\mathrm{x}$-ray optical systems for proper focusing. The necessary optics cannot be fabricated without the use of precise optical metrology instrumentation. In particular, the Long Trace Profiler (LTP) based on the pencil-beam interferometer is a valuable tool for low-spatial-frequency slope measurement with x-ray optics. The limitations of such a device are set by the amount of systematic errors and noise. A significant improvement of LTP performance was the addition of an optical reference channel, which allowed to partially account for systematic errors associated with wiggling and wobbling of the LTP carriage. However, the optical reference is affected by changing optical path length, non-homogenous optics, and air turbulence. In the present work, we experimentally investigate the questions related to the use of a precision tiltmeter as a reference channel. Dependence of the tiltmeter performance on horizontal acceleration, temperature drift, motion regime, and kinematical scheme of the translation stage has been investigated. It is shown that at an appropriate experimental arrangement, the tiltmeter provides a slope reference for the LTP system with accuracy on the level of $0.1 \mu \mathrm{rad}(\mathrm{rms})$.
\end{abstract}

Keywords: tiltmeter, slope measuring instrument, long trace profiler, LTP, error reduction, reference, X-ray optics, optical metrology

\section{INTRODUCTION}

With the growing requirements for the design and fabrication of high-performance x-ray optical systems comes a need for more precise metrology, and thus improvements in the accuracy of metrology instruments. This involves the suppression and elimination of sources of systematic error and noise in slope measuring devices, in particular, the LTP, which is used as a basic metrology tool for characterizing an X-ray optic figure with slope variation on the level of $\sim 0.5-1 \mu_{\text {rad. }}{ }^{1-4}$ The LTP optical schematic is a realization of the pencil-beam interferometer. In the interferometer, two parallel light beams, possessing a phase difference, are made to interfere at the focus of a Fourier transform lens. The resulted interference fringe pattern, recorded with a position-sensitive detector placed at the focus, has two strongly marked peaks with a minimum between the peaks. The position of the minimum is a measure of slope of the mirror surface at the place of the beam reflection. The LTP records the local slope profile of a surface by measuring the reflection angle of a laser sample beam as the beam is transported across the surface by an air bearing carriage. Due to the translation of the optical sensor, the LTP has a unique capability for surface figure metrology of very long, metersize, mirrors. However, non-idealities of the translation mechanism as well as non-idealities of the optical elements bring forth a number of systematic effects, which often dominate over the random noise of the LTP measurement. ${ }^{5,6}$

A significant improvement to the LTP schematic was made by the addition of a reference arm to record (simultaneously with the sample beam) angle variations of a reference beam reflected from a stationary reference mirror. ${ }^{7,8}$ By subtracting the reference output from the sample channel, the effects of laser pointing instability and changes in tilt due to mechanical movement of the carriage are significantly reduced. Unfortunately, use of a reference beam in an LTP has some limitations. Particularly, the optical path length of the reference beam does not match the sample beam, and undergoes change based on distance of the LTP carriage from the reference mirror. In order to combine in one reference arm the errors due to the laser pointing instability and carriage motion, an additional optical element, a Dove prism, was added. Moreover, the reference beam has a large unshielded optical path through the air that is subject to air-convection noise. ${ }^{9}$ A detailed discussion of sources of the LTP systematic errors and random noise can be found, e.g., in Ref. ${ }^{10}$, where a new method of calibration of slope measuring instruments is proposed.

*JLKirschman@lbl.gov; tel +1-510-486-4117; fax +1-510-486-7696 
In the present article, we discuss a possible radical solution of the reference channel problems mentioned above. That is, to replace the previously described reference channel with a high-precision tiltmeter for independent monitoring of the carriage wiggling and wobbling. The Dove prism can be removed and the reference mirror can be placed on the carriage in order to continue monitoring for laser pointing instability. A tiltmeter references to the gravity vector and has no optical path limitations; thus, it is unaffected by air convection. In such an arrangement, the LTP optical head can be fully shielded and temperature stabilized inside an enclosure.

Below, we investigate the major properties of a tiltmeter, Applied Geomechanics Model $755-1172,{ }^{11}$ for use as an LTP reference (Sec. 2). In Sec. 3 we present data on the characterization (using a tiltmeter) of the performance of two linear translation stages, one the current LTP-II at the ALS Optical Metrology Laboratory (OML), and another one based on a Nano-motion drive specially developed for the next generation of the OML LTP. Design and efficiency of an active temperature stabilization developed for the tiltmeter is presented in Sec. 4. A cross-check of the reference signals simultaneously measured with the LTP-II optical reference arm and with the tiltmeter-based reference channel is given in Sec. 5.

\section{CHARACTERISTICS OF TILTMETERS}

The basic design of the type of tiltmeters ${ }^{11}$ used in this work consists of a gas bubble suspended in electrolytic fluid between 2 exciting electrodes and a pick-up electrode. The gas bubble orientates itself in relation to the gravity vector; thus, a tilt results in reorientation of the bubble and a change of the AC resistance between the electrodes. This is then amplified by internal circuitry and output as a DC voltage, which can be converted to a change of angle by a scaling factor subject to calibration. The level of the output noise was verified to be on the level of $0.1 \mu \mathrm{rad}$. The total range of the device is \pm 16 milliradians, though in actual use, the tiltmeters were manually aligned to be as close to 0 tilt as possible ( $<1 \mathrm{mrad}$ ) in order to avoid linearity errors. Note that because the tiltmeter is to be used in the reference channel, the overall useful range is significantly small. The range is determined by the slope variation of the LTP ceramic beam and amplitude of wiggling and wobbling of the carriage, which are typically less than $\sim 20 \mu \mathrm{rad}$. Therefore, relative calibration accuracy of $\sim 0.5 \%$ is suitable for an LTP measurement with accuracy of $\sim 0.1 \mu \mathrm{rad}$.

First, we found the tiltmeter to be extremely sensitive to a variation of the surrounding temperature. Figure 1 shows the dependence of the tiltmeter output signal on temperature variation in the ALS OML. ${ }^{12}$ The environmental conditions in the lab are supported with an air conditioning system. The overall temperature consists of a 120 mK total temperature decrease in the late afternoon and night over the course of 12 hours, and a periodic fluctuation due to the air conditioning system on/off cycles on the level of $\sim 20 \mathrm{mK}$. Note that experiments were done in a plastic-enclosed hutch used to house the Developmental Long Trace Profiler (DLTP) set-up, an LTP under design.
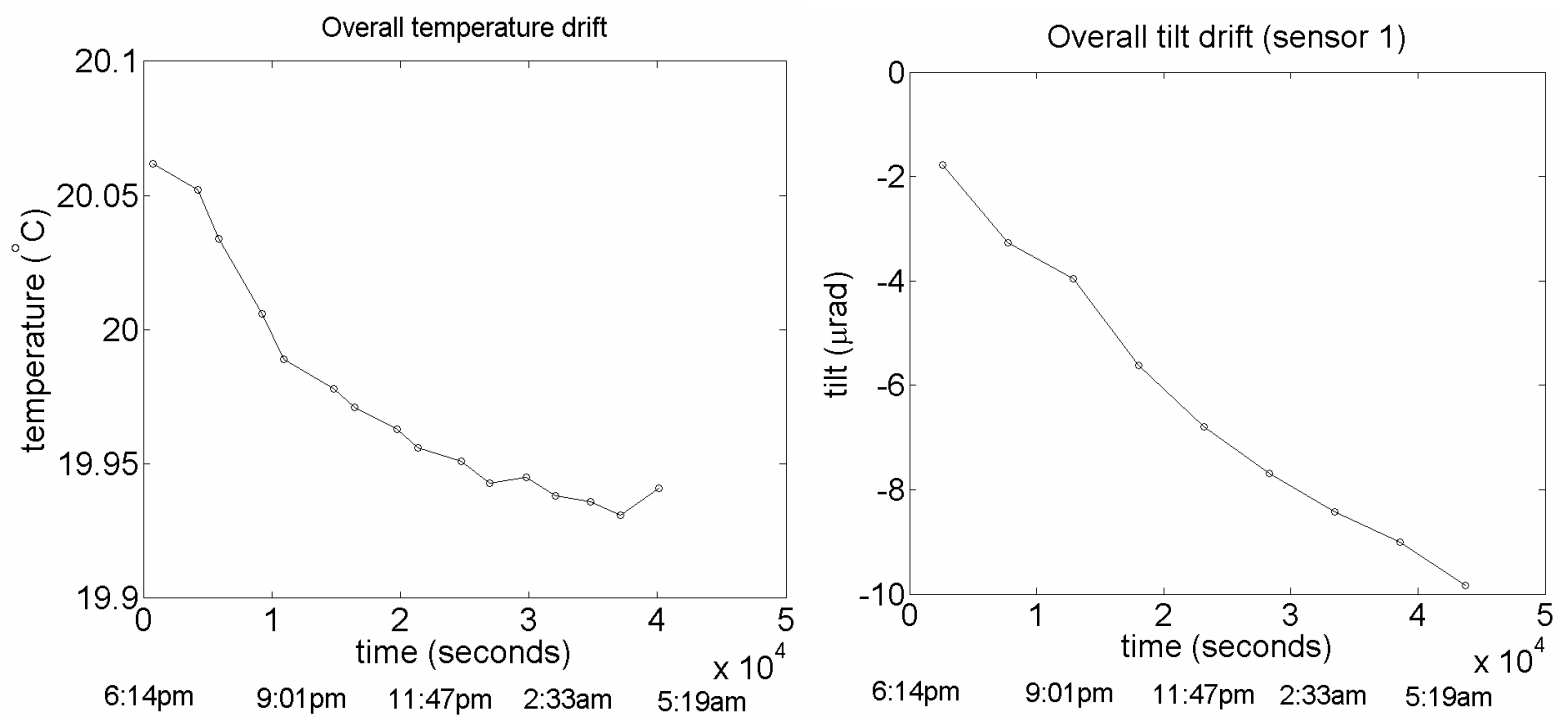

Figure 1: Temperature drift (left) and the corresponding reading from the tiltmeter (right) in the OML over a 12 hour period of time. 
For the measurement in Fig. 1, the temperature sensor located in the DLTP hutch is an integrated-circuit-based sensor (National Semiconductor LM35) with resolution of about $2 \mathrm{mK}$. The sensor is connected through an Applied Geomechanics 781 Signal Conditioning Unit to be used in conjunction with the testing of the tilt sensors. The tilt sensor was mounted on the center of an optical table next to the temperature sensor.

Figure 1 shows a long-term, 12 hour period of temperature change in the OML, and the corresponding output signal of the tiltmeter during this time. The total tilt reading change was approximately $9 \mu \mathrm{rad}$, which is less than $1 \mu \mathrm{rad} / \mathrm{hour}$. If one attributes the drift to the corresponding temperature change inside the hutch of $\sim 120 \mathrm{mK}$, this gives an estimation for the temperature coefficient of the sensors of $\sim 80 \mu \mathrm{rad} / \mathrm{K}$ for very slow temperature variations.

On the same optical table as the tiltmeters, a linear stage based on air-bearing suspension and Nano-motion drivers is also assembled (see Fig. 2). The stage is a part of the DLTP set-up, and it is designed for translating a carriage with LTP optics along a ceramic beam for approximately one meter distance. The stage is driven by piezoceramic ultrasonic motors, and possesses a linear encoder with specified accuracy of $\sim 0.1$ microns. While measuring the temperature dependence of the tiltmeter, both tilt sensors were mounted on the optical table. The temperature sensor was attached to the optical table between the sensors (Fig. 2). In order to reproduce the real environmental conditions inside the hutch, the stage was activated in such a way that the carriage traversed a distance of $900 \mathrm{~mm}$ along the ceramic beam in $2 \mathrm{~mm}$ increments (every 5.7 seconds).

The result of the measurements is shown in Fig. 3. The sensitivity of the tiltmeters was enough to pick up the gradual change of tilt of the optical table in response to the changing weight distribution due to carriage movement, traces (a) and (b) in Fig. 3. It is also notable that small fluctuations in the tiltmeter outputs, traces (c) and (d), correlate directly with the on/off cycles of the air conditioner unit, which can be seen in Fig. 3 (e). The $\sim 20 \mathrm{mK}$ peak-to-peak fluctuation of temperature results in a $\sim 0.6 \mu$ rad change of tilt reading. The ratio of the amplitude of the periodical tilt reading variations and the variation of temperature provides the dynamical temperature coefficient of the tiltmeter to be approximately 30-40 $\mu \mathrm{rad} / \mathrm{K}$. This value significantly differs from the one estimated from the long-term drift. It can be due to the large time constant for the sensor's temperature reaction to a change of temperature of the optical table. Due to the large time constant, the effect of the relatively fast temperature variation is averaged. Moreover, a phase shift between the temperature oscillation and oscillation of the tiltmeter output signal seen in Fig. 3 can be also attributed to the large time constant.

The performed investigations demonstrate that the periodical fluctuation of temperature, characteristic for the current OML environmental conditions, significantly impacts the tiltmeter measurements. In Sec. 4, we describe the performance of the tiltmeter mounted inside a thermo-insulating enclosure with active temperature stabilization. In this case, the effect of the environmental temperature fluctuations to the performance of the tiltmeter is significantly suppressed.

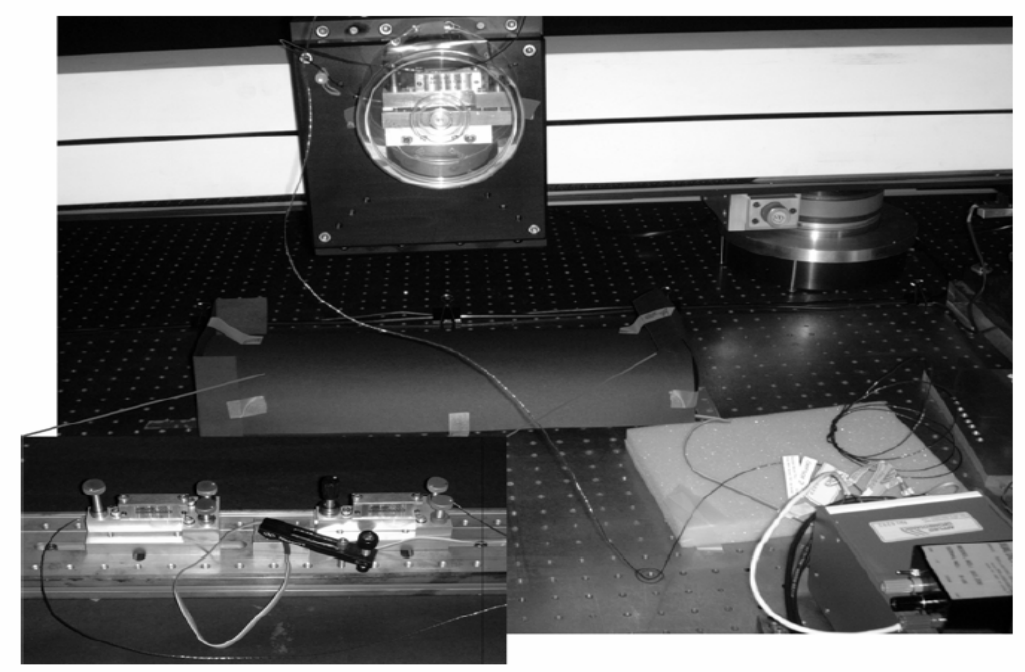

Figure 2: Image of setup with tiltmeters and temperature sensor for temperature experiments (inset), and with a tiltmeter mounted on the DLTP carriage. 


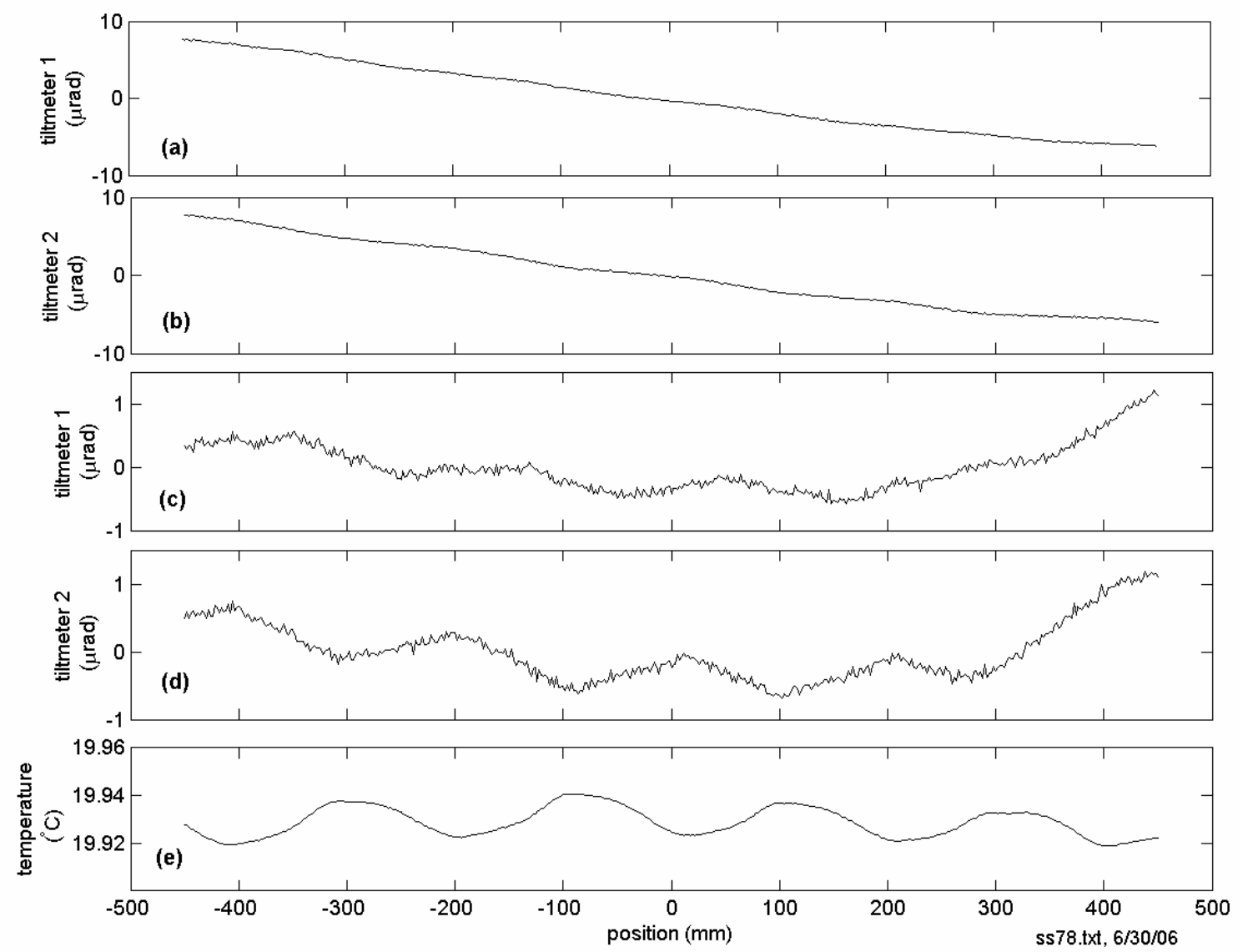

Figure 3: The tilt of the table as the carriage incrementally translates across $900 \mathrm{~mm}$ distance is shown in (a) and (b) corresponding to each tiltmeter. (c) and (d) show the same output after a best fit linear subtraction to remove the tilt for each tiltmeter respectively. The fluctuations correspond to the 10 minute cycling of the air conditioner as in (e), which is on the order of $20 \mathrm{mK}$.

The bubble suspended in fluid approach which comprises the tiltmeters also was found to be susceptible to acceleration. According to a technical note from the manufacturer, for small angles, acceleration in horizontal direction directly corresponds to a tilt. ${ }^{13}$ The recommendation from the company for overcoming this was to enable the 2-pole Butterworth low-pass filter built into the signal conditioner. However, this was not found to be effective. Moreover, the filter has an integration time of 7.5 seconds, which is longer than the desired sampling rate for most LTP systems including the DLTP set-up and the LTP-II recently upgraded at the ALS OML. ${ }^{14}$

In order to characterize the tiltmeter response to acceleration, we mounted the tiltmeter on the carriage of the DLTP (Fig. 2). Basically, the DLTP linear translation stage provides relatively smooth/accurate motion through $900 \mathrm{~mm}$ of travel. ${ }^{15}$ However, there is a starting/stopping acceleration with magnitude and duration dependent on the preset parameters of the Nano-motion drivers. For the measurements shown in Fig. 4, the DLTP carriage was set for continuous motion movement at a high velocity with different accelerations. Velocity was set to $20 \mathrm{~mm} / \mathrm{sec}$ and acceleration to $1 \mathrm{~mm} / \mathrm{sec}^{2}, 0.5 \mathrm{~mm} / \mathrm{sec}^{2}$, and $0.1 \mathrm{~mm} / \mathrm{sec}^{2}$. These resulted in tilt signals of about $100 \mu \mathrm{rad}, 50 \mu \mathrm{rad}$, and $10 \mu \mathrm{rad}$, respectively. Thus, the obtained result suggests a linear relationship between acceleration and the corresponding output signal of the tiltmeter.

The perturbations of tiltmeter measurements due to the acceleration can be significantly suppressed if a start-stopmeasurement regime of the LTP measurements is used. Such a regime was implemented in the DLTP setup (Sec. 3) and in the upgraded LTP-II ${ }^{14}$ (Sec. 4). 


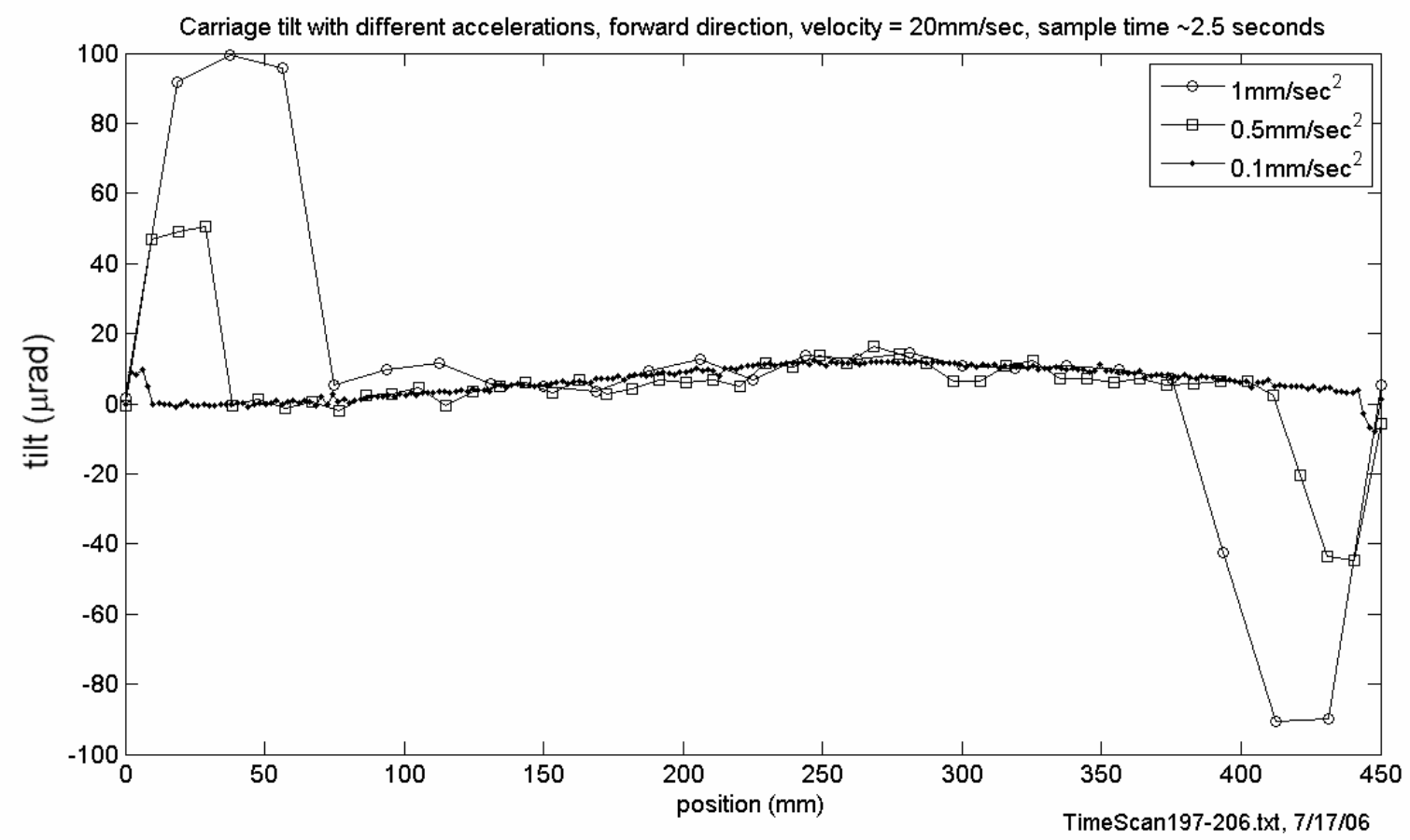

Figure 4: Tiltmeter output showing the effect of acceleration and deceleration on tilt. The tilt corresponds directly to the level of acceleration set for each translation of the carriage. Sampling is done every 2.5 seconds.

\section{TILTMETER-BASED ANALYSIS OF CARRIAGE MOVEMENT}

The tiltmeter allows us to characterize the movement of a carriage in terms of smoothness of movement. For example, several movement regimes for the DLTP carriage were analyzed with use of the tiltmeter mounted on the carriage. For the DLTP, two movement schemes were evaluated. The first is a constant speed measurement in which data is recorded while the carriage is continuously in motion. The second is a start-stop-measurement regime in which the carriage stops every $2 \mathrm{~mm}$, pauses for about 3 seconds (which gives time for the carriage and tiltmeter to settle), then takes a measurement.

For constant motion (Fig. 5), it was found that higher velocities significantly increased noise in tiltmeter measurements. A velocity of $1 \mathrm{~mm} / \mathrm{sec}$ resulted in noise on the level of $\sim 1 \mu \mathrm{rad}$ (rms). A reduction in velocity to $0.33 \mathrm{~mm} / \mathrm{sec}$ yielded 0.1..0.15 $\mu \mathrm{rad}$ (rms) of noise. Additionally, there was a notable dependence on the direction of the carriage. Movement in the reverse direction (from right to left) at $1 \mathrm{~mm} / \mathrm{sec}$ was smoother and produced approximately $0.5 \mu \mathrm{rad}$ (rms) noise. This nonhomogeneity is believed to be due to overall tilt of the ceramic beam, and thus different loads on the Nanomotion drive in opposite directions. Changing the distribution of weight on the table can lead to noise (probably friction-based) across certain areas of the beam, due to off-axis tilting of the beam. The noise is partially correctable when decreasing the velocity of the carriage from $1 \mathrm{~mm} / \mathrm{sec}$ to $0.33 \mathrm{~mm} / \mathrm{sec}$, resulting in the same noise in both directions. However, there was an area of about $100 \mathrm{~mm}$ in one direction which had increased noise approximately 7 times the noise for the rest of the measurement. Careful balancing of the carriage, along with alignment of the ceramic beam and loads on the table, should partially remedy this problem.

A very important limitation to using tiltmeters in a continuous motion regime is the motor control and response for the carriage. As it attempts to reach a certain position, small accelerations can lead to a change in tiltmeter output, depending on the setting of the feedback loop for the motor and encoder. It is thus only practical to use tiltmeters in continuous motion when the mirror to be measured does not require highly accurate measurements or on a carriage which is extremely finely tuned and moves at a constant velocity smoothly. An example of how this can significantly limit use of the tiltmeter is our experience with the conventional LTP-II before the upgrade. ${ }^{14}$ In this case (Fig. 6), continuous movement of the carriage results in a very large (> $20 \mu \mathrm{rad}$ ) fluctuation of the tiltmeter output signal, in spite 
of the fact that the LTP optical reference arm shows a regular, less than 1 rrad (rms) fluctuation. This is due to how the LTP carriage is moved via a drive belt with lots of constant acceleration/deceleration.
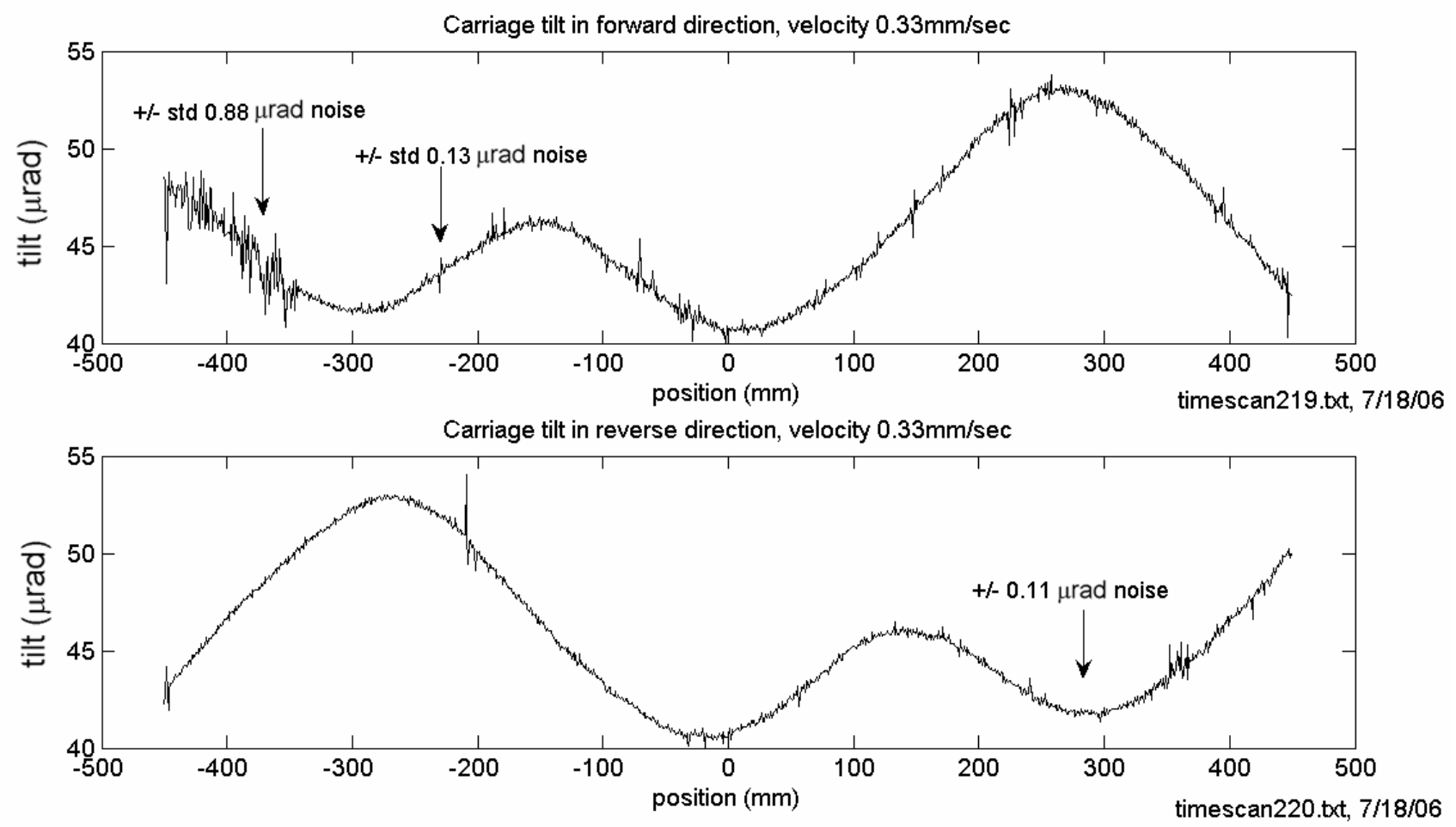

Figure 5: Tiltmeter output for DLTP setup in forward (top) and reverse (bottom) movement direction. The areas of fluctuation are related to small changes in acceleration likely due to the balance of the beam, loads on the picomotors, and motor tuning parameters.
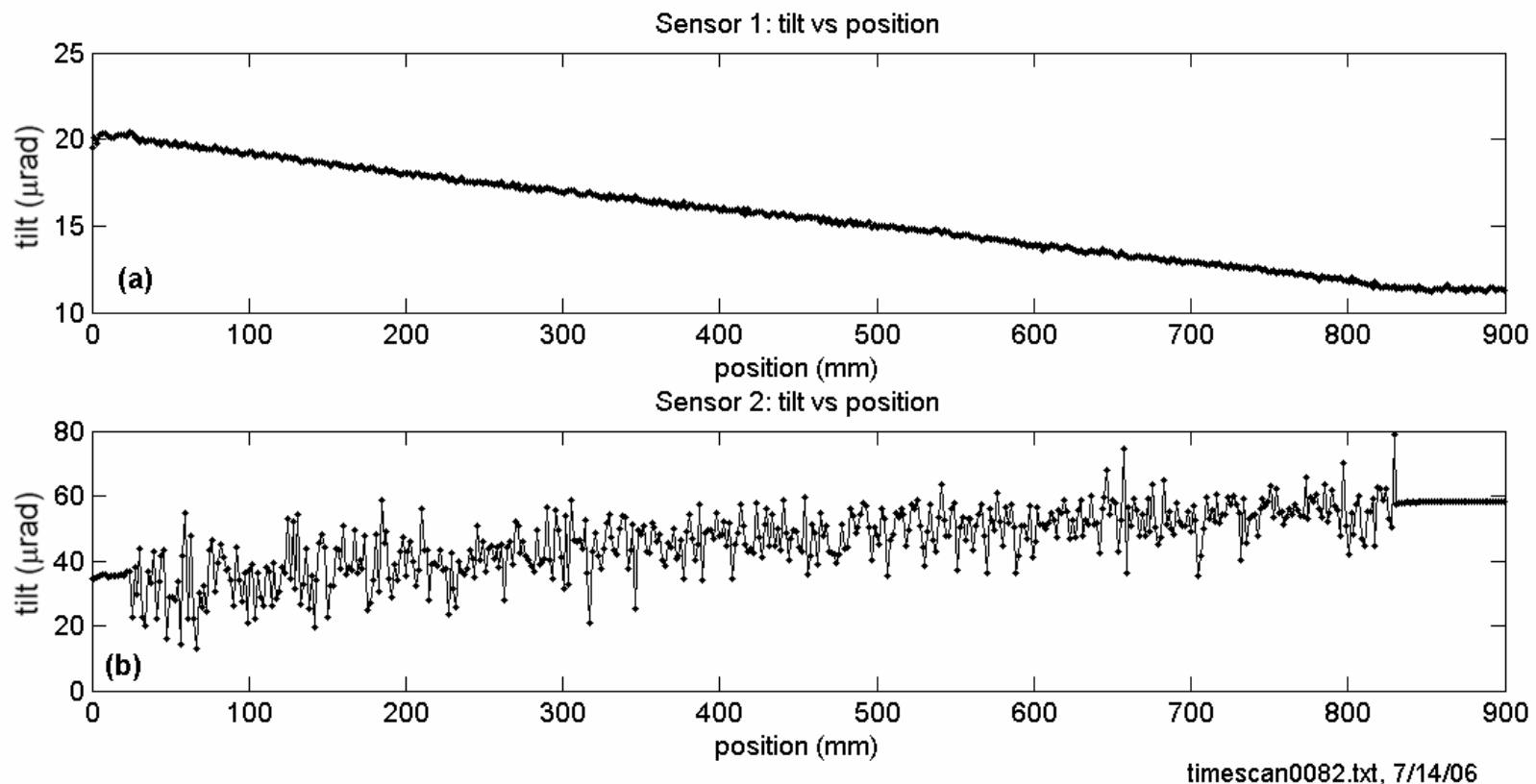

Fig. 6. Tiltmeter output for pre-upgrade LTP-II setup in forward direction. The tilt of (a) sensor 1 (on table) is very smooth, but (b) sensor 2 (on carriage) is extremely noisy due to horizontal acceleration/deceleration of the LTP-II carriage in continues motion mode. 
Figure 7 shows the measurements with the DLTP stage in start-stop-measurement mode. In this case, high velocities ( $20 \mathrm{~mm} / \mathrm{sec}$ ) resulted in noise of around $0.3 \mu \mathrm{rad}(\mathrm{rms})$. A velocity of $1 \mathrm{~mm} / \mathrm{sec}$ (suitable for metrology applications) resulted in noise of 0.1-0.15 $\mu \mathrm{rad}$. There was no indication of directional dependence or added noise due to off-axis tilt. Start-stop measurement mode is still affected by the level of acceleration due to the acceleration and deceleration at every $2 \mathrm{~mm}$ of translation, but can be corrected by allowing the carriage time to settle after a move before taking data. By decreasing the acceleration to $1 \mathrm{~mm} / \mathrm{sec}^{2}$, noise for start-stop-measurement mode was reduced to less than $0.1 \mu \mathrm{rad}$ (rms). Therefore, both motion regimes can be used with the DLTP to detect slope changes of the carriage. However, start-stop measurement mode allows the greatest accuracy and repeatability. With the LTP system in continues motion mode, it is not possible to use the tiltmeter due to the nature of the motor and drive system.
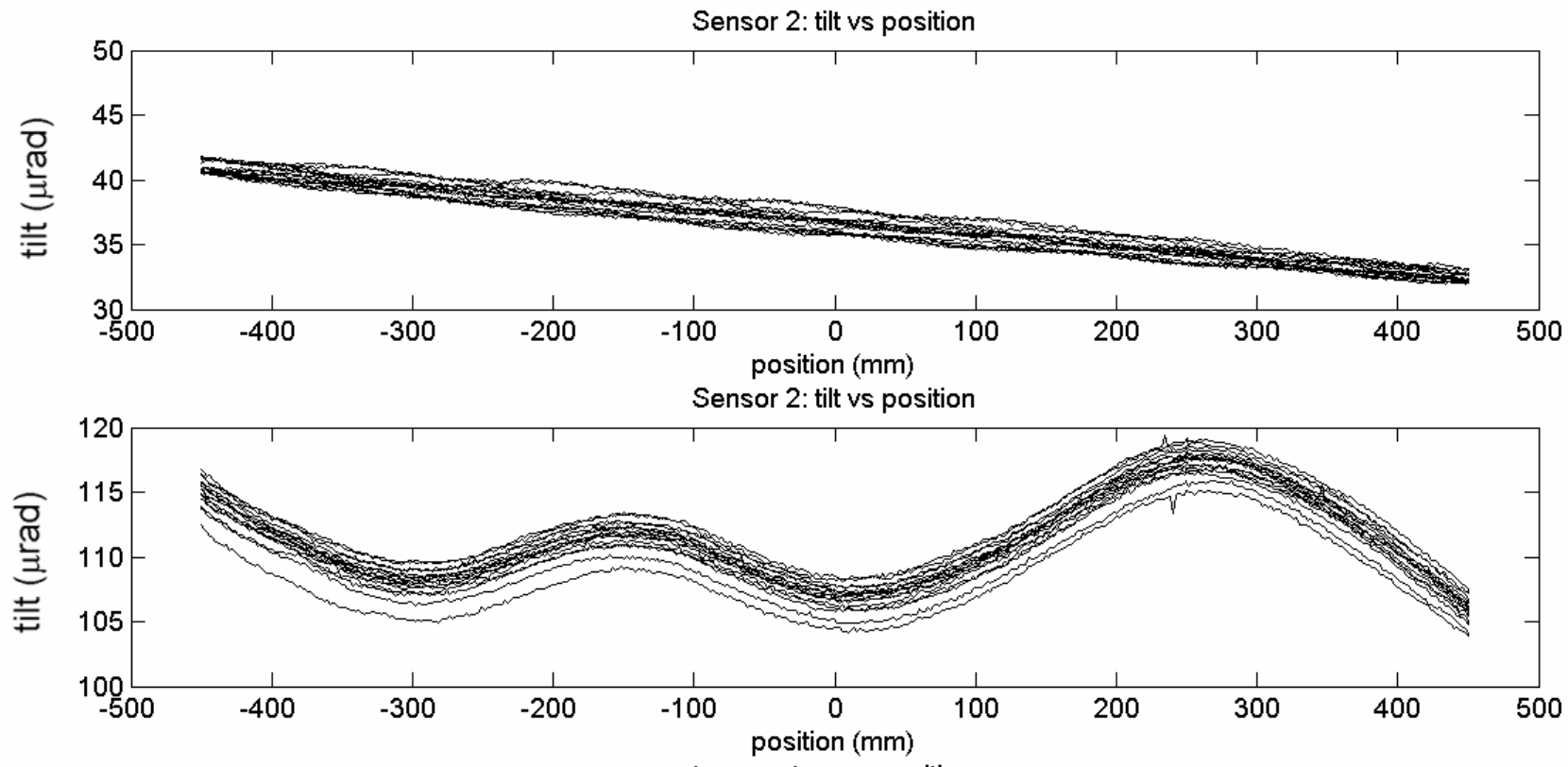

temperature vs position

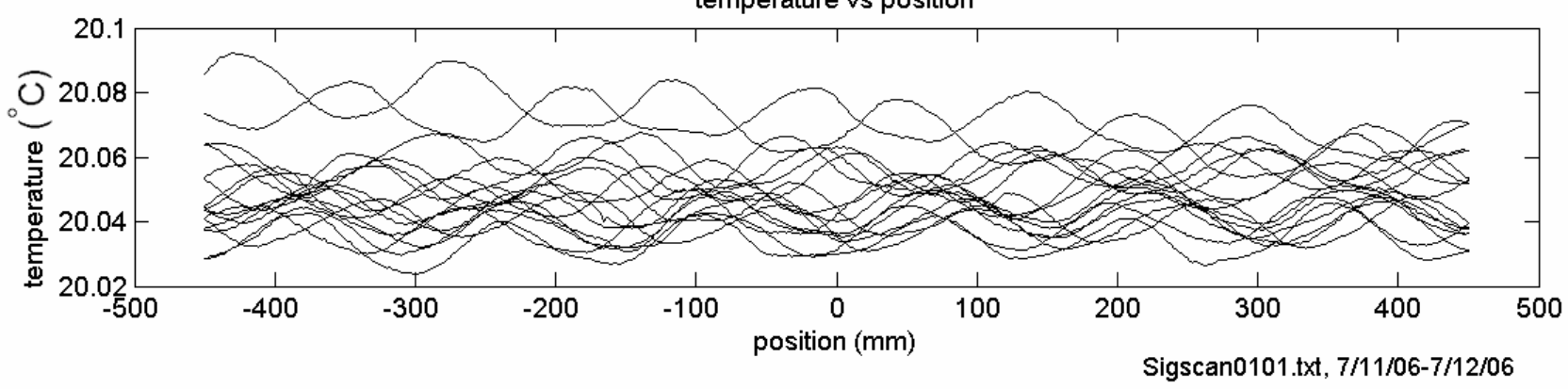

Figure 7: Tiltmeter output for DLTP setup with a tiltmeter on the table (top), one on the carriage (middle), and a temperature sensor (bottom), set to traverse back and forth 8 times over the course of several hours. The shape of the beam and tilt of the table are very repeatable, with a drift due to the temperature.

\section{PERFORMANCE OF TILTMETER WITH ACTIVE TEMPERATURE STABILIZATION}

Based on the above analysis in Sections 2 and 3, in order to implement tiltmeters as a reference channel, a thermoinsulating enclosure with active temperature stabilization was developed. Figure 8 shows the design of the enclosure with a cover removed. For the temperature stabilization we use a Peltier TEC element with a fan to dissipate the power. A temperature sensor (AD590) provides the feedback signal to a temperature controller. ${ }^{16}$ 


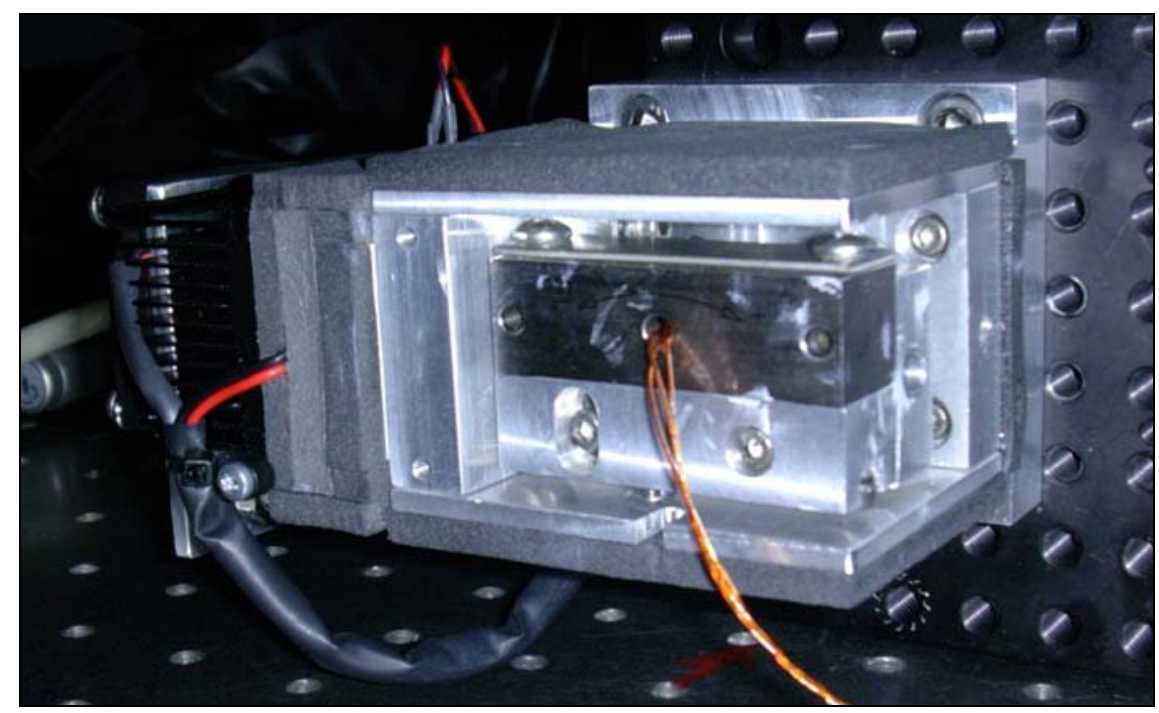

Figure 8: Tiltmeter active temperature stabilization enclosure. Fan, heatsink, and Peltier cooler are on the left, with a temperature feedback sensor indicated by the uppermost wires. The tiltmeter itself (with the copper wires) is suspended by several adjustable mounting pieces used for initial alignment.
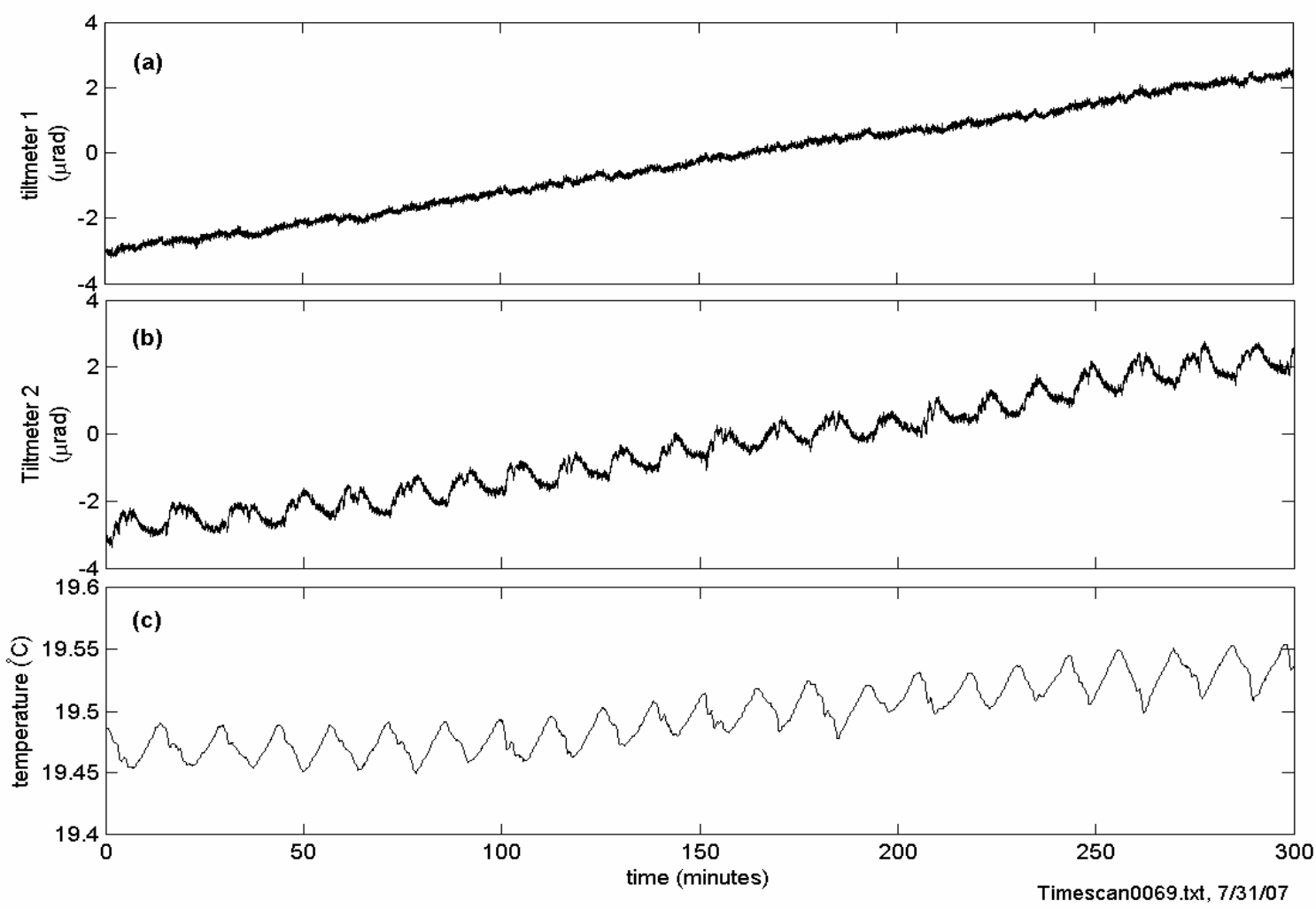

Figure 9: Illustration of the efficiency of the active temperature stabilization enclosure developed for the tiltmeter sensor. Tilt sensor 1 (a) is in the enclosure, tilt sensor 2 (b) is on the optical table in the open along with a temperature sensor (c). The enclosure helps to suppress the output signal variations associated with the periodical change of the ambient temperature by a factor of approximately 5 . However, there is still a notable drift, likely due to temperature drift of the conditioning electronics, along with other possibilities such as thermal expansion of the table and surrounding materials. 
The efficiency of the temperature stabilization system for suppression of the effect of environmental temperature variation was tested with two tilt sensors mounted on the DLTP optical table. One sensor was placed inside the temperature stabilized enclosure (Fig. 8); another one was mounted open as it is shown in Fig. 2. In order to increase the outside temperature variability, the DLTP hutch was kept open. The result of the comparison measurements is shown in Fig. 9. The enclosure helps to suppress the output signal variations associated with the periodical change of the ambient temperature by a factor of approximately 5 .

While the effects of the periodical change in temperature are suppressed as indicated in Fig. 9, there is also a steady drift which is the same in both tiltmeter outputs. This leads to the possibility that temperature fluctuations also have an effect on the conditioning electronics. Alternatively, another temperature-related effect could also be responsible, such as thermal expansion in the table and related materials. However, since the drift is the same in both tilt outputs, it is possible to subtract out the drift to get a differential output. This is subject to further investigation, and it emphasizes the need for very stable environmental conditions.

\section{TILTMETER APPLICABILITY AS A REFERENCE CHANNEL FOR LTP}

As we have mentioned, our upgraded LTP-II is operating in start-stop measurement mode. ${ }^{14}$ This opens a possibility to use an additional reference channel based on a precise tiltmeter. In order to verify the applicability of the tiltmeter discussed above for such a reference channel, we carried out an experiment to cross-check the tiltmeter measurements with the measurements from the existing LTP optical reference arm. In the course of the experiment, the tiltmeter (without temperature stabilization) was mounted on the LTP carriage. Another sensor was mounted on the table in order to monitor the tilt of the LTP optical table due to the carriage translation. The measurements were made when the carriage was moved over $900 \mathrm{~mm}$ of travel, to reflect the length of a significantly long mirror. The LTP hutch was closed to decrease the temperature variation inside the hutch. In the same manner as typical measurements with the upgraded LTP, to allow time for the carriage to settle before data is taken, a 3 second delay is set between each move. The results of this test are shown in Figs. 10 and 11.

Figure 10 shows the measurement of tilt of the carriage of the upgraded LTP-II ${ }^{14}$ with the two tiltmeter sensors. The difference of the measurements (trace (c) in Fig. 10) represents the tilt trace corresponding to the carriage tilting during translation as it should be seen with the LTP optical reference channel using a reference mirror mounted on the optical table. The amplitude of the temperature fluctuation inside the hutch was measured to be about $\pm 5 \mathrm{mK}$ which is small enough not to cause any noticeable temperature effect on the second tilt sensor.

Figure 11 provides a comparison of the slope trace obtained with the conventional LTP reference arm (trace (a) in Fig. 11) with the carriage tilt trace (trace (c) in Fig. 10 and trace (b) in Fig. 11) measured with two tiltmeter sensors. The difference of the measurements with the LTP optical and the tiltmeter-based reference channels (Fig. 11 (c)) is rather small [ $0.8 \mu \mathrm{rad}$ (rms)], but still significantly larger than the desired LTP accuracy of $\sim 0.1 \mu \mathrm{rad}$ (rms). However, only a very tiny part of the difference can be attributed to the temperature effect on the second tilt sensor. A more probable explanation is that the systematic error of the optical reference channel increases with increasing distance between the reference mirror and the LTP optical head. Note also a significantly smaller (by a factor of more than 3) random noise of the trace measured with the tiltmeters.

\section{CONCLUSION}

We have investigated the possibility of replacing the conventional LTP reference channel with a high-precision tiltmeter for the independent monitoring of the carriage wiggling and wobbling. The performed investigations have suggested that the tiltmeter ${ }^{11}$ can be used to provide a reference channel independent of the position of the carriage or from air convection noise. For the tiltmeter sensor, we have developed a special thermal insulating enclosure with active temperature stabilization. The enclosure allows us to efficiently suppress (by factor of $\sim 5$ ) periodical temperature effects to the tiltmeter measurements, though there is still a need for temperature stabilization of related electronics and of the overall environmental conditions. We have also demonstrated that the sensitivity of tiltmeter to horizontal acceleration can be overcome if one uses a reasonably slow start-stop measurement mode of LTP operation. This allows, in principle, LTP measurements on the level of $0.1 \mu \mathrm{rad}$, to be possible. 

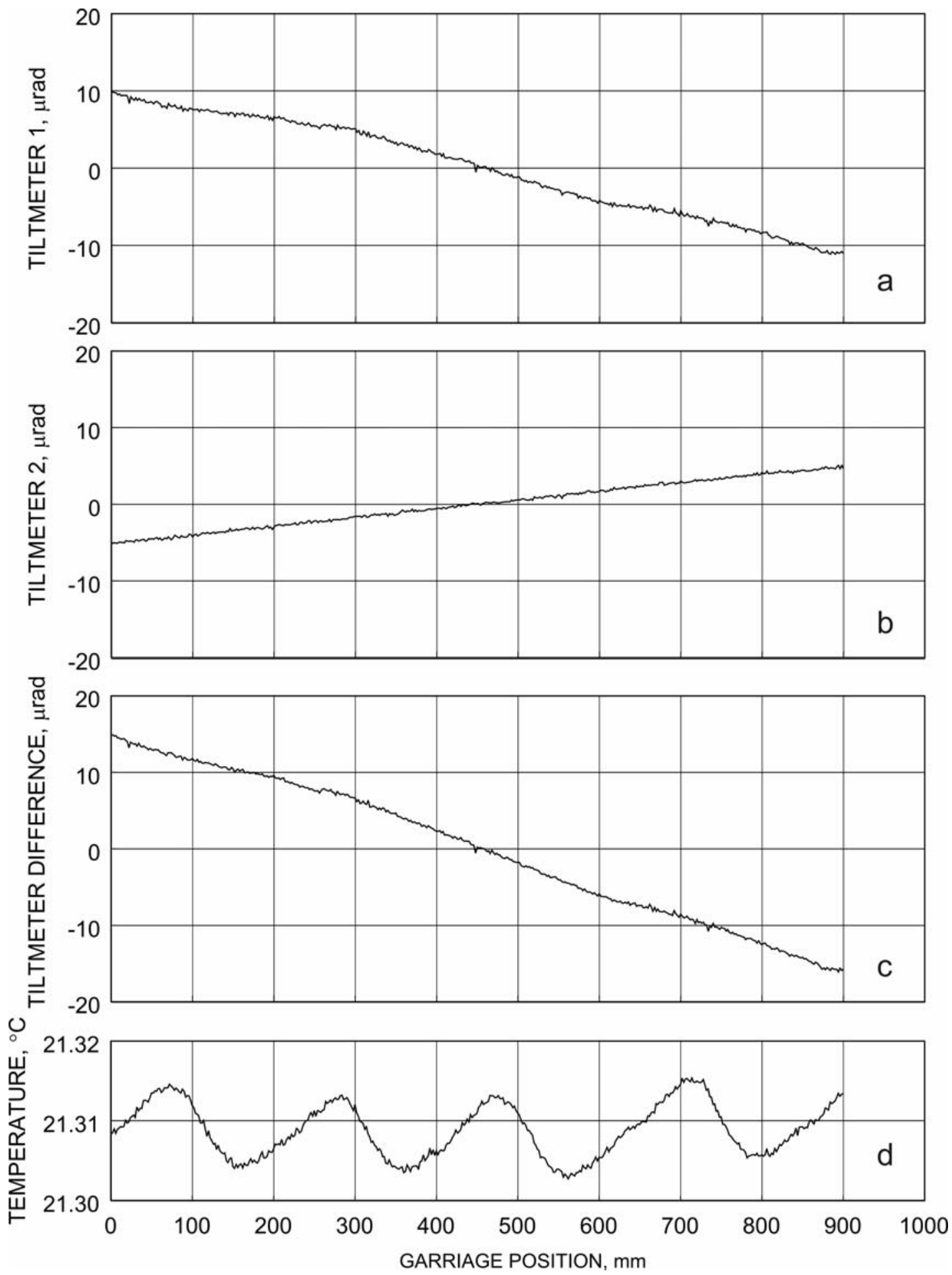

Figure 10: Measurement of tilt of the carriage of the upgraded LTP-II ${ }^{14}$ with two tiltmeter sensors, the first sensor placed on the carriage (a) and the second one mounted on the LTP optical table (b). The difference of the measurements (c) represents the tilt trace corresponding to the carriage tilting during translation. The amplitude of the temperature fluctuation (d) inside the hutch is about $\pm 5 \mathrm{mK}$, which is small enough not to cause any noticeable temperature effect on the second tilt sensor. 

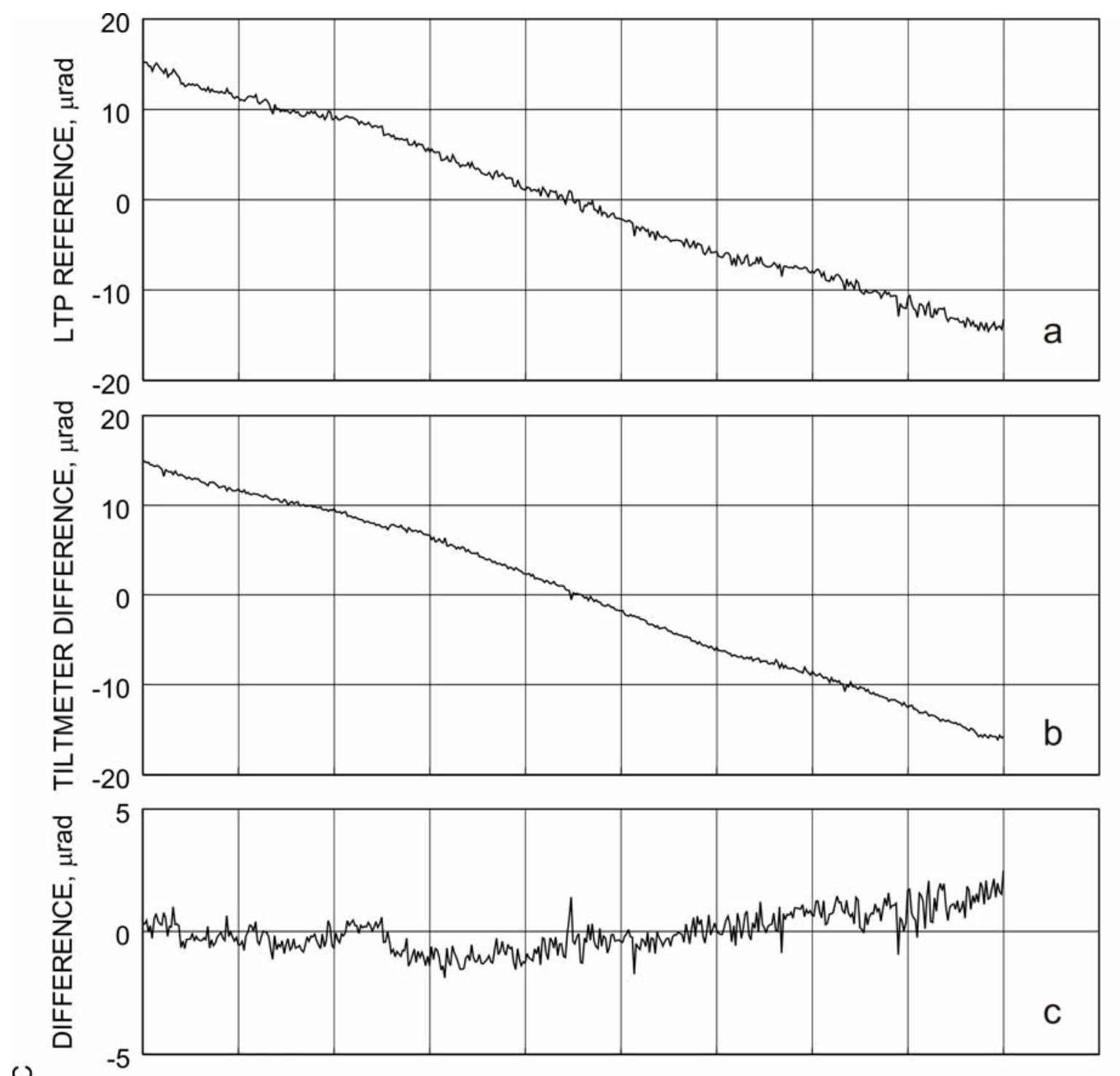

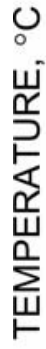

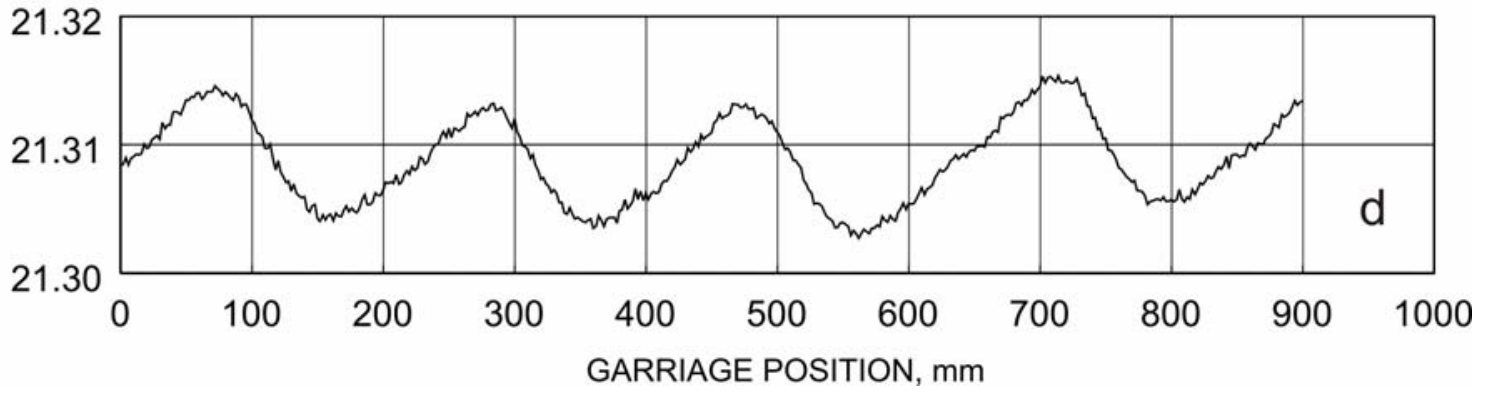

Figure 11: Comparison of the slope trace obtained with the conventional LTP reference arm (a) with the tilt trace (b) measured with two tiltmeters corresponding to the carriage tilting during translation (compare with Fig. 10 (c)). The difference of the measurements with the LTP optical and the tiltmeter-based reference channels are depicted with trace (c). The trace of temperature fluctuation inside the hutch (d) is shown to have a very tiny correlation with the differential trace (c). 
Note that in the course of the measurements described, we used the tiltmeter calibration provided by the manufacturer. The calibration assumes an absolute linearity of the sensor. In principle, even for a very small range of interest (about $\pm 20 \mu \mathrm{rad}$ ), the calibration can be nonlinear. A comparison with a well-calibrated auto-collimator or other angle measurement device is desirable. The corresponding experiments are in progress at the ALS OML.

\section{ACKNOWLEDGEMENTS}

The authors are grateful to Alastair MacDowell, Wayne McKinney, Howard Padmore, and Tony Warwick for extremely useful discussions. This work was supported by the U. S. Department of Energy under contract number DEAC02-05CH11231.

\section{DISCLAIMER}

Certain commercial equipment, instruments, software, or materials are identified in this document. Such identification does not imply recommendation or endorsement by the US Department of Energy, LBNL or ALS nor does it imply that the products identified are necessarily the best available for the purpose.

\section{REFERENCES}

1. E. L. Church, P. Z. Takacs, "Use of an optical profiling instrument for the measurement of the figure and finish of optical quality surfaces,” Wear, 109, 241-57 (1986).

2. $\quad$ P. Z. Takacs, Shinan Qian, J. Colbert, “Design of a long trace surface profiler,” Proc. SPIE 749 (1987), 59-64.

3. P. Z. Takacs, S. K. Feng, E. L. Church, Shinan Qian, W-M. Liu, "Long trace profile measurements on cylindrical aspheres,” Proc. SPIE 966, 354-64 (1989).

4. S. C. Irick. W. R. McKinney, “Advancements in one-dimensional profiling with a long trace profiler,” Proc. SPIE 1720, 162-8 (1992).

5. V. V. Yashchuk, "Positioning Errors of Pencil-beam Interferometers for Long Trace Profilers," Proc. SPIE 6317, 63170A (2006).

6. V. V. Yashchuk, S. C. Irick, and A. A. MacDowell, "Elimination of 'ghost'-effect-related systematic errors in metrology of x-ray optics with a long trace profiler,” Proc. SPIE 5858, 58580X (2005).

7. S. C. Irick, W. R. McKinney, D. L. Lunt, P. Z. Takacs, "Using a straightness reference in obtaining more accurate surface profiles from a long trace profiler (for synchrotron optics),” Rev. Sci. Instrum. 63(1) , 1436-8 (1992).

8. S. C. Irick, "Improved measurement accuracy in a long trace profiler: compensation for laser pointing instability," Nucl. Instrum. Meth. A 347(1-3), 226-30 (1994).

9. V.V. Yashchuk, S.C. Irick, A.A. MacDowell, W.R. McKinney, and P.Z. Takacs, "Air convection noise of pencilbeam interferometer for long trace profiler,” Proc. SPIE 6317, 63170D (2006).

10. V. V. Yashchuk, W. R. McKinney, T. Warwick, T. Noll, F. Siewert, T. Zeschke, R. D. Geckeler, "Proposal for a Universal Test Mirror for Characterization of Slope Measuring Instruments," Proc. SPIE 6704, 6704-9 (2007) this volume.

11. Applied Geomechanics 755-1172 Miniature Tilt Sensors (http://www.geomechanics.com/dspproduct.cfm?prid=1).

12. J. L. Kirschman, R. S. Celestre, S. C. Irick, T. Warwick, B. V. Smith, E. E. Domning, V. V. Yashchuk, “Temperature Stability in the Optical Metrology Laboratory,” ALS Beamline Note, LSBL-798, (2006).

13. Applied Geomechanics Technical Note: “Acceleration Effects on Tiltmeter Measurements" (http://www.geomechanics.com/dspapp.cfm?appid=85).

14. J. L. Kirschman, E. E. Domning, K. D. Franck, S. C. Irick, A. A. McDowell, W. R. McKinney, G. Y. Morrison, B. V. Smith, T. Warwick, V. V. Yashchuk, "Flat-field calibration of CCD detector for Long Trace Profiler," SPIE Proc. 6704-18 (2007) - this volume.

15. J.L. Kirschman, E.E. Domning, S.C. Irick, A.A. McDowell, G.Y. Morrison, B.V. Smith, V.V. Yashchuk, "Characterization of Linear Translation Stage for Developmental Long Trace Profiler," ALS Beamline Note, LSBL-798 (2006).

16. Thorlabs TED 200 Laser Diode Temperature Controller (http://thorlabs.com/Thorcat/7600/7619-D02.pdf). 